\title{
CENTENARIO DE LA ADORACIÓN DELOS REYES MAGOS EN EL BARRIO DE SAN BLAS
}

Yvanna Betshabe Núñez Esquivel ${ }^{1}$, Raúl Nicolás Castro Triveño ${ }^{2}$.

\section{RESUMEN}

El acto sacramental es una composición dramática en la que se describen escenas de la Historia Sagrada, a través de personajes alegóricos, en el barrio de San Blas donde los habitantes actúan demostrando su amor y religiosidad simulando la adoración de los Reyes Magos en la navidad al niño Jesús, esta escenificación cumple 100 años este años. Se realizó un estudio descriptivo donde se realizó entrevistas a los vecinos del barrio y a los organizadores de la adoración, se tiene como resultado muestra de la cultura y tradición de una fiesta realzada por los habitantes cusqueños del barrio de San Blas, se tiene que este festival de los reyes magos cumple con diversas actuaciones y etapas donde se hace $\mathrm{n}$ muestra de la tradición y representación al aire libre como una educación popular religiosa.

PALABRAS CLAVE: Adoración, Navidad, Reyes Magos.

\begin{abstract}
The sacramental act is a dramatic composition in which are described scenes of the Sacred History, through allegorical characters in the San Blas neighborhood where habitants act demonstrating their love and religiosity simulating the adoration of the Wizard Kings to the child Jesus in Christmas. This dramatization turns 100 years in this year and descriptive study was carried out interviewing to the neighbors of the neighborhood and to the organizers of worship obtaining as result a sample of the culture and tradition of a celebration realized by the habitants of Cusco in the neighborhood of San Blas. This celebration of the Three Wizard Kings has various performances and stages where they are a tradition show and an outdoor representation of popular religious education.

KEYWORDS: Worship, Christmas, Three Kings.

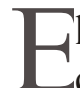
auto sacramental es una composición dramática en la que se
\end{abstract}

1 Ingeniera Civil.

2 Arquitecto, bachiller en Ingeniería Civil. Docentes de la Escuela de Topografia de la Universidad Nacional de San Antonio Abad del Cusco. E-mail: rnicolascastrot@gmail.com 
describen escenas de la Historia Sagrada a través de personajes alegóricos, para su gran difusión y representación al aire libre, con eminentes fines de educación popular religiosa. Surgió en España en el Siglo XVII y llego a América apenas iniciada la Invasión Europea, siendo uno de los géneros más cultivados dentro del movimiento teatral en la Colonia, de esta manera se instaura en nuestra ciudad este tipo de escenificaciones con la integración y aportes andinos, siendo entre estos "La Adoración de los Reyes Magos"

"En ella se recitan y cantan canciones en quechua al Cusco, Los temas son situaciones cotidianas del pueblo con cierta dosis de pedagogía y jocosidad que culminan con la merienda de estilo y la qhaswa."

El estudio realizado es descriptivo mediante una serie de análisis de vivencias y entrevistas con los vecinos de San Blas es así que en el Cusco en el barrio de San Blas el sacerdote cusqueño Juan Francisco Palomino Cáceres (1873 - 1949) fue Canónigo Teologal de la Catedral del Cusco, párroco de San Blas por más de cincuenta años, prolífico escritor sagrado, excelso predicador y cultor del Quechua, y sobre todo hombre de bien y gran sensibilidad social, conocido como el famoso "Papacha" Palomino.

"Por esta vocación a la fe católica quiso llegar a las mayorías populares, sobre todo de su amadísimo barrio de San Blas - T'oqo Kachi o "hueco de sal", uno de los principales barrios incas, haciendo conocer los misterios de la religión. Para ello preparo autos sacra-mentales, coloquios y misterios, como es el caso del auto sacramental "La Adoración de los Reyes Magos", instaurado el año de1916. Desde entonces, hasta este año se celebrara el centenario, se lo representa en la plaza de San Blas en horas de la tarde del tercer domingo de diciembre (aunque algunos años el cuarto, por su proximidad a la navidad), siendo "la más elaborada y la más duradera de su tipo y una de las pocas representaciones con parlamento y verso cantado en español y quechua, que sobreviven de la tradición dramática del Auto

Sacra-mental español en territorio sur andino del Perú".

Se tiene referencias que a inicios del siglo pasado aún se realizaba en el barrio de Belén una representación parecida, aunque mucho más simple. Posiblemente Palomino se basó en dicha versión, enriquecida luego por su pluma con textos y parlamentos quechuas, en su afán de adecuarla de mejor manera al sentir popular.

Finalmente hasta le agrego una última parte costumbrista y algo más: los primeros años se iniciaba "la Fiesta" nombre familiar con que los Sanbleños conocen la adoración- con canciones y poemas patrióticos, ante la presencia de la madre patria y las Cautivas Tacna y Arica, personajes simbólicos en clara alusión a la reciente e infausta Guerra del Pacifico.

La "Adoración de los Reyes Magos", o también conocida como la "Degollación de los Santos Inocentes", comprende cuatro secciones, con una duración total de tres horas, siendo las siguientes: 


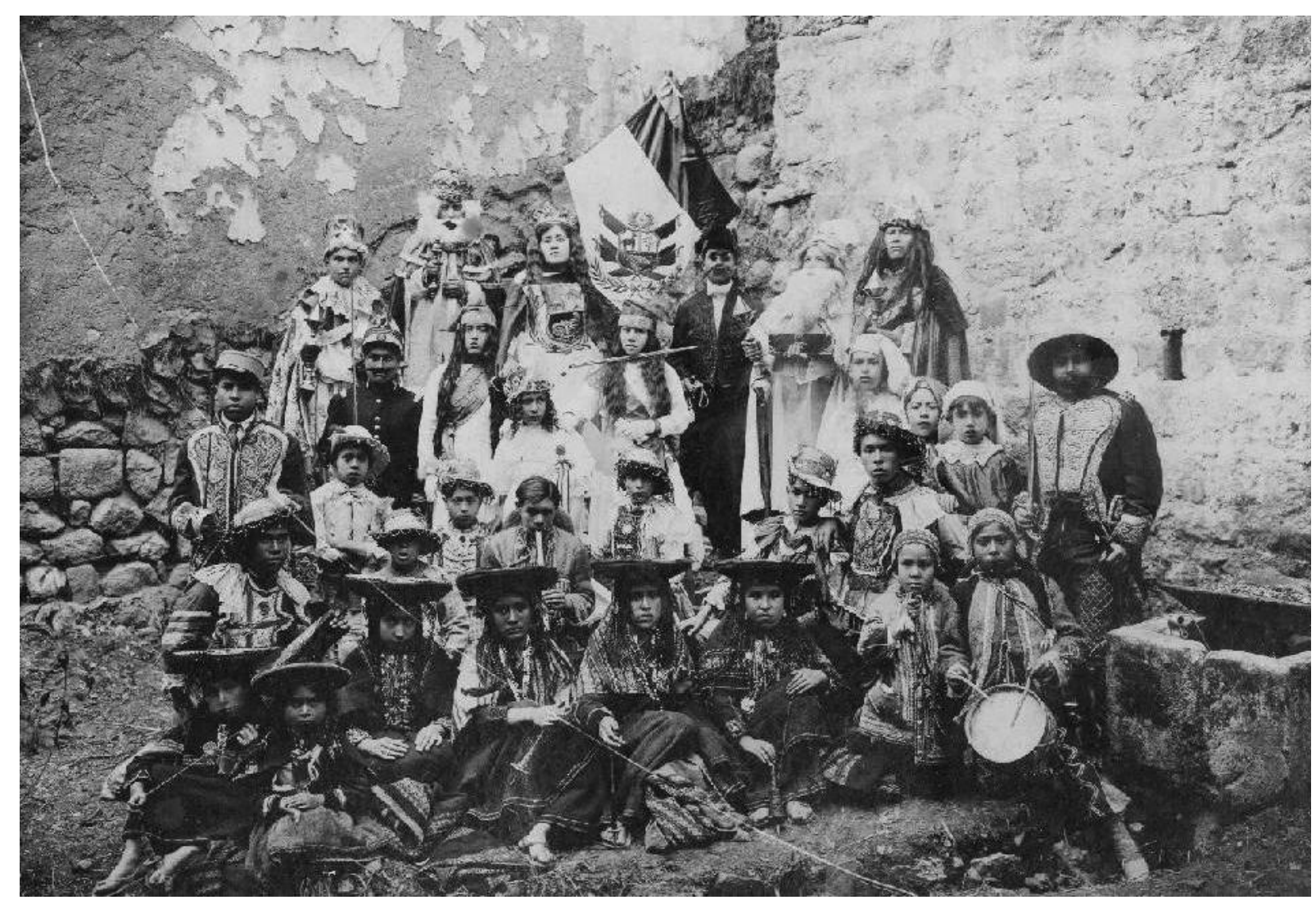

Adoración de los Reyes Magos en San Blas, 1921.

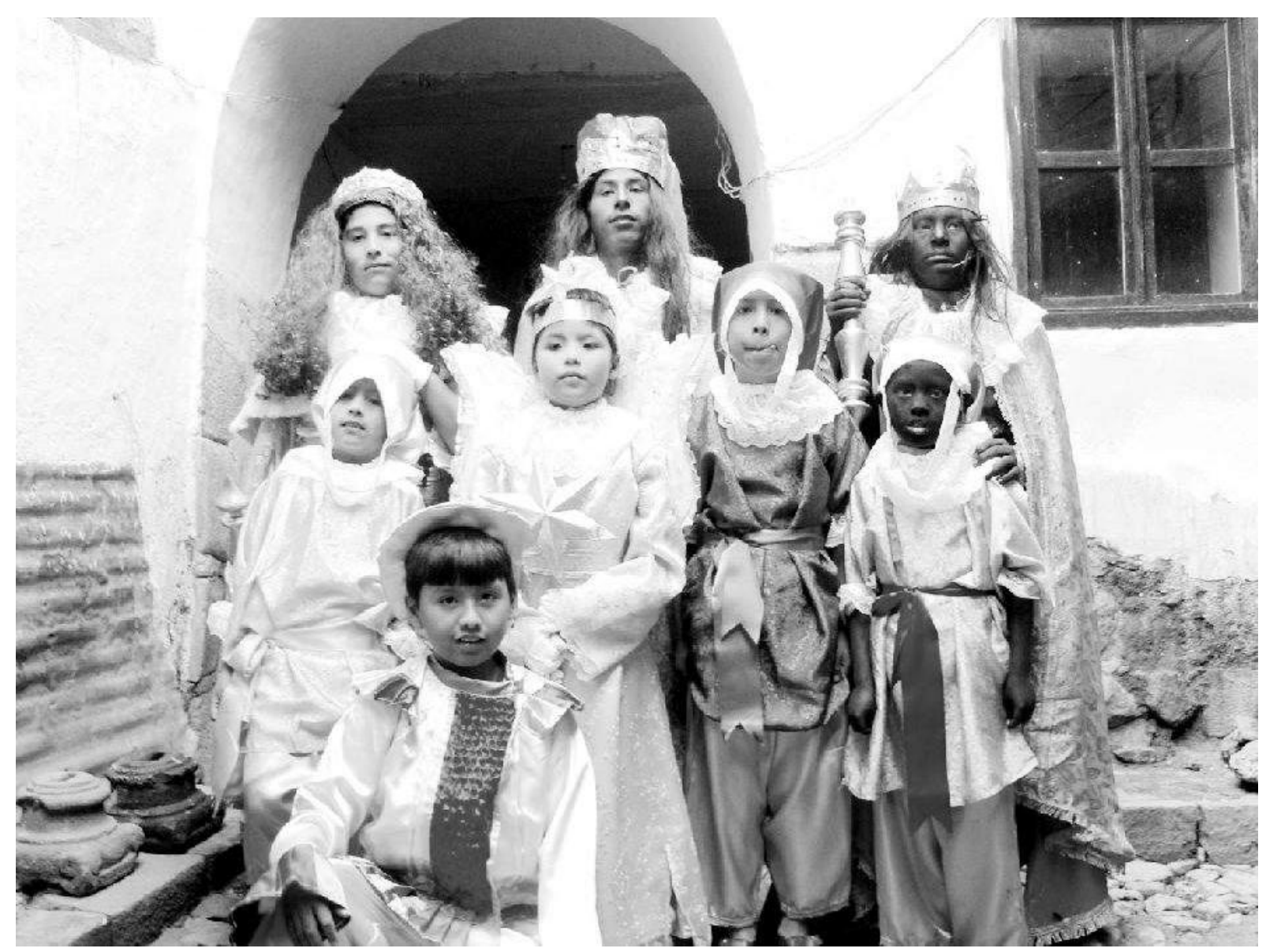

Adoración de los Reyes Magos en el patio de la familia Rosas en San Blas, 1921. 
-Adoración de Los Reyes Magos.

Sección principal de la

representación que se desarrolla

en diferentes partes de la plaza de

San Blas teniendo como centro de atención el "Balcón del Rey

Herodes" (esquina de la plaza con

la cuesta de San Blas), y el

tabladillo delante del templo, el

"Portal de Belén" con un gran telón de fondo de un nacimiento cusqueño que cubre la puerta del templo, es toda la escenificación de como los tres Reyes del Oriente llegan al portal de Belén pasando por la entrevista con Herodes y el encuentro con la estrella refulgente y su adoración al Niño Jesús con sus recitaciones y regalos y luego con la orientación del Ángel para su vuelta a casa.

-Degollación de Los Santos Inocentes Corta escena, aunque de mucha importancia, que se desarrolla delante del templo.

Tres intrépidos vasallos del Rey Herodes irrumpen entre el público buscando a los "niños recién nacidos, hasta los dos años" para ser decapitados, según el mandato de Herodes. Seis criaturas (hechas de paja y sangre de anilina en tripas de res), arranchadas a sus madres son decapitadas en el escenario y arrojadas sangrantes al público, que festeja el acontecimiento. Baile De Los Villancicos Sanbleños Los villancicos son canciones cortas y sencillas de origen también español, del siglo XVI, que se identifican sobre todo con el espíritu navideño, siendo su carácter eminentemente popular. En el Cusco algunos villancicos aún conservan rasgos de su origen, pero la gran mayoría son ya de corte andino, en ritmos de huayno, pasacalle o qhaswa, sazonados con letras en castellano yquechua, aunque algunos solo musicales. En la adoración se cantan y bailan 14 villancicos, a cargo de una cuadrilla de diez parejas de niños "Pastorcitos de San Blas", con trajes y sombreros multicolores, siendo las dos últimas de ch'utis (niños indígenas) con sus típicas vestimentas regionales. Los dirige un caporal, ya un tanto mayor, mientras que el ángel que los guía participa alegremente de las diversas coreografías. Entre danza y danza, un pastorcito recita una loa al niño Jesús. Estampa Costumbrista. Posiblemente como un resabio del teatro quechua tan en boga en la región a inicios del siglo XX. Juan Francisco Palomino agrego a la adoración una última parte costumbrista, realmente alejada del tema navideño, que sin embargo confiere a la actividad en conjunto un carácter eminentemente popular y festivo en ella se recitan y cantan canciones en quechua al Cusco, Los temas son situaciones cotidianas del pueblo con cierta dosis de pedagogía y jocosidad que culminan con la merienda de estilo y la qhaswa.

En el presente, la adoración de los reyes magos se sigue festejando el tercer domingo de diciembre continuando con la tradición popular y cultura de nuestra ciudad, en este centenario, la adoración de los reyes magos perdura por la animosidad y la ayuda de los vecinos de San Blas, pero más aún por la familia que ha mantenido esta tradición brindando su casa y su esfuerzo para realizar esta festividad, "la familia Rozas", esta festividad costumbrista debería ser reconocida como patrimonio cultural viviente por el Ministerio de Cultura.

\footnotetext{
REFERENCIAS

Beyersdorff Margot, "La adoración de los Reyes Magos", Cusco, Centro de Estudios Rurales Andinos Bartolomé de las Casas. 1988.

Valencia Espinoza, Abraham, "Personajes legendarios del barrio de San Blas", El Sol del Cusco, 19 de diciembre DE 2004
} 